\title{
Pneumococcal neuraminidase substrates identified through comparative proteomics enabled by chemoselective labeling
}

\author{
Janet E. McCombs and Jennifer J. Kohler \\ Department of Biochemistry, The University of Texas Southwestern Medical Center, Dallas, TX \\ 75390-9038, USA
}

\section{Abstract}

\begin{abstract}
Neuraminidases (sialidases) are enzymes that hydrolytically remove sialic acid from sialylated proteins and lipids. Neuraminidases are encoded by a range of human pathogens, including bacteria, viruses, fungi, and protozoa. Many pathogen neuraminidases are virulence factors, indicating that desialylation of host glycoconjugates can be a critical step in infection. Specifically, desialylation of host cell surface glycoproteins can enable these molecules to function as pathogen receptors or can alter signaling through the plasma membrane. Despite these critical effects, no unbiased approaches exist to identify glycoprotein substrates of neuraminidases. Here, we combine previously reported glycoproteomics methods with quantitative proteomics analysis to identify glycoproteins whose sialylation changes in response to neuraminidase treatment. The two glycoproteomics methods - periodate oxidation and aniline-catalyzed oxime ligation (PAL) and galactose oxidase and aniline-catalyzed oxime ligation (GAL) - rely on chemoselective labeling of sialylated and non-sialylated glycoproteins, respectively. We demonstrated the utility of the combined approaches by identifying substrates of two pneumococcal neuraminidases in a human cell line that models the blood-brain barrier. The methods deliver complementary lists of neuraminidase substrates, with GAL identifying a larger number of substrates than PAL (77 versus 17). Putative neuraminidase substrates were confirmed by other methods, establishing the validity of the approach. Among the identified substrates were host glycoproteins known to function in bacteria adherence and infection. Functional assays suggest that multiple desialylated cell surface glycoproteins may act together as pneumococcus receptors. Overall, this method will provide a powerful approach to identify glycoproteins that are desialylated by both purified neuraminidases and intact pathogens.
\end{abstract}

\section{Introduction}

Mammalian cells are covered in a dense outer layer of glycoconjugates, many of which include terminal sialic acids. Neuraminidases, or sialidases, are enzymes that hydrolytically remove sialic acids from sialylated protein and lipid substrates, thereby altering their localization, activity, and interaction events. A wide array of pathogens, including bacteria,

*jennifer.kohler@utsouthwestern.edu.

Supporting Information

Detailed descriptions of methods, lists of protein substrates identified, verification of glycosylation inhibitors in hCMEC/D3 cells, localization of proteins identified in each proteomics trial, validation of identified proteins, assessment of sialylation in hCMEC/D3 cells, and knockdown verification. This material is available free of charge via the Internet at http://pubs.acs.org. 
viruses, fungi, and protozoa, produce neuraminidases. ${ }^{1-4}$ Many of these neuraminidases cleave sialic acid from host cell surfaces to facilitate infection. ${ }^{2,5}$ Neuraminidase-mediated alterations in the glycosylation states of host cell surface molecules can dramatically affect host-pathogen interactions as well as both inter- and intra-cellular signaling events in host cells. ${ }^{6-8}$ In this way, pathogen neuraminidases often operate as virulence factors. Therefore, identifying host-cell substrates of pathogenic neuraminidases could provide details on mechanisms of pathogen infections.

One such pathogen that utilizes neuraminidases in infection is Streptococcus pneumoniae (pneumococcus), which afflicts millions of adults and children worldwide. ${ }^{9}$ Notably, pneumococcus contributes to over $50 \%$ of bacterial meningitis cases, which are often fatal infections. ${ }^{10}$ Though treatments exist, vaccines only target a subset of serotypes and there is an alarming resistance to antibiotics. ${ }^{11}$ Thus, there persists a need to better understand mechanisms underlying pneumococcal virulence in meningitis. Importantly, pneumococcus utilizes over ten glycosidases, enzymes that modify cell surface glycans, as virulence factors to enable host infection. ${ }^{12,8}$ Of these, three are neuraminidases (NanA, NanB, and NanC). ${ }^{13}$ Indeed, cleavage of sialic acid by neuraminidases is an initial event, enabling subsequent cleavages of glycan linkages by other glycosidases. ${ }^{14,15}$ This process of glycan remodeling has been shown to promote pneumococcal host-microbe interactions, uncover host factors utilized by the pneumococcus to facilitate infection, and could even aid pneumococcal evasion of host immune responses. ${ }^{8}$

To date, NanA has been shown to work in conjunction with two other glycosidases to reveal a receptor on epithelial cell surfaces to which pneumococci bind. ${ }^{14,16}$ In addition, both NanA and NanB are essential in mouse models of infection. ${ }^{17,18}$ Importantly, knockout of NanA results in the inability of pneumococci to adhere to or invade brain microvascular endothelial cells, suggesting a key role for NanA in pneumococcal meningitis infections and crossing of the blood-brain barrier. ${ }^{19,20}$ While NanA and NanB are found in all or most serotypes of pneumococci, respectively, $\mathrm{NanC}$ is present in only about half. ${ }^{13}$ As of yet, no function has been described for $\mathrm{NanC}$, but, intriguingly, NanC-expressing pneumococci are isolated primarily from cerebral spinal fluid, suggesting $\mathrm{NanC}$ may play a pivotal role in pneumococcal meningitis infections. ${ }^{13}$ While it is clear neuraminidases play key roles in pneumococcal infections, the identities of their host protein substrates remain unknown. Identification of such factors would elucidate mechanisms of pneumococcal infection, potentially revealing targets for new therapeutics.

The substrate specificity of neuraminidases is driven primarily by recognition of the glycan, and not the protein, portion of a glycoprotein. However, by altering the glycosylation of host proteins, neuraminidases can have a profound effect on protein function, location, and activity. Therefore, elucidation of the glycoproteins that are neuraminidase substrates could highlight specific pathways that neuraminidases modulate in the host cell, which are then utilized by pathogens for efficient infection. However, unbiased methods to discover the protein component of neuraminidase substrates do not exist. Given the hydrolytic nature of neuraminidases, capturing their exact substrates is challenging. Here we make use of two methods for labeling glycans on living cells with biotin in a chemoselective manner using galactose oxidase and aniline-catalyzed oxime ligation (GAL) and periodate oxidation and 
aniline-catalyzed oxime ligation (PAL). ${ }^{21,22}$ First, cells treated with neuraminidase or not are labeled using either GAL or PAL before capturing proteins by streptavidin purification. Purified proteins are then identified by mass spectrometry and analyzed using a semiquantitative comparative proteomics method (SINQ) ${ }^{23}$ Finally, we perform a comparative analysis to identify host glycoproteins whose glycosylation is altered by neuraminidase activity. By using both PAL and GAL in this method, we obtained complementary lists of neuraminidase protein substrates. We performed these analyses on a human cell line that models the blood-brain barrier to gain comprehensive information about pneumococcal neuraminidase substrates in this physiologically-relevant cell type, as a step toward identifying mechanisms through which this pathogen causes infection in host tissues.

\section{Results and Discussion}

Given the role of glycans in pneumococcal infection, we first determined whether inhibiting biosynthesis of specific classes of glycans affected adherence of bacteria to human cells. We chose to use the human cerebral microvascular endothelial cell line (hCMEC/D3), ${ }^{24}$ an established model of the blood-brain barrier, to enable identification of neuraminidase substrates potentially involved in meningitis infections. Cells were cultured for $72 \mathrm{~h}$ with kifunensine, an inhibitor of maturation of N-linked glycans, ${ }^{25}$ benzyl-a-GalNAc, a decoy substrate that interferes with GalNAc-type O-linked glycosylation, ${ }^{26}$ or 3Fax-peracetyl Neu5Ac (3Fax), a metabolic inhibitor of sialylation. ${ }^{27}$ To confirm that inhibitors functioned as expected, we measured the binding of specific lectins to hCMEC/D3 cell surfaces. In particular, cells treated with kifunensine displayed the expected increased binding of the lectin Concanavalin A (ConA), which binds to immature high mannose structures (Supplementary Figure 1a). Culturing cells with benzyl-a-GalNAc led to increased binding of $\mathrm{T}$ antigen by peanut agglutinin (PNA), as observed in other cell lines (Supplementary Figure 1b). ${ }^{28-30}$ Finally, binding of Maackia amurensis lectin II (MAL II), a lectin that specifically recognizes a2-3 linked sialic acids, was decreased in cells treated with 3Fax (Supplementary Figure 1c), indicative of decreased sialylation on the cell surface.

Once inhibition of various glycan classes was validated, we treated inhibited cells with pneumococci for 30 min to determine whether the amount of bacteria adhered to cells was altered by changes in glycan expression. While the $\mathrm{N}$-linked modulator kifunensine appeared to have no effect on bacterial binding, we found that inhibition of O-linked glycan maturation by benzyl-a-GalNAc led to an increase in bacterial adherence to the cell (Figure 1a). This result is consistent with a study demonstrating that removal of core 1 structures by a pneumococcal O-glycosidase contributes to host colonization and adherence to epithelial cells. ${ }^{15}$ Finally, treatment with 3Fax, which decreases cell-surface sialylation, also resulted in an increase in pneumococcus binding. This is to be expected, as the pneumococcal neuraminidases NanA and NanB have been shown to be virulence factors, ${ }^{17,18}$ indicating removal of sialic acids is important in infection. Indeed, when we treated cells with NanA, a known virulence factor, or the less studied NanC, followed by incubation with pneumococci, binding of bacteria to hCMEC/D3 cells increased (Figure 1b). Therefore, both NanA and NanC appear to remove sialic acids from underlying structures utilized by pneumococcus for binding. 
Modifications in glycosylation can have a large impact on the function, location, and activity of cell surface glycoproteins. Given the essential nature of neuraminidases in pneumococcal infection, we therefore postulated that identification of the proteins they modify could be useful in determining mechanisms through which pneumococci cause infection. We set out to develop an assay for determining specific protein substrates of neuraminidases in our model cell line, hCMEC/D3. Interactions between hydrolytic enzymes, such as neuraminidases, and their substrates are transient in nature, making such identifications a challenging task. We therefore desired to label specific glycan structures in both untreated and neuraminidase-treated cells, allowing comparison between samples to identify proteins desialylated by pneumococcal neuraminidases. We pursued two complementary approaches, each relying on chemoselective chemistries that have long been used in labeling specific glycan structures, ${ }^{31,32}$ and have been made more effective in the past decade: periodate oxidation and aniline-catalyzed oxime ligation (PAL; Figure 2a) and galactose oxidase and aniline-catalyzed oxime ligation (GAL; Figure 2b). ${ }^{21,22}$ In PAL, sialic acids are oxidized by periodate, creating an aldehyde at the $\mathrm{C} 7$ position, which can then be selectively labeled through an oxime ligation to an aminooxy-conjugated biotin. Similarly, in GAL, oxidation of terminal galactose (Gal) and $N$-acetylgalactosamine (GalNAc) residues at the C6 position by galactose oxidase produces an aldehyde that can again be selectively labeled through an oxime ligation. Thus, both PAL and GAL labeling are sensitive to changes in sialylation.

To determine whether we could label cells in a neuraminidase-dependent manner, we compared hCMEC/D3 cells left untreated or treated with NanA or NanC for $1 \mathrm{~h}$ at $37^{\circ} \mathrm{C}$ before labeling with PAL or GAL. NanA is the only non-specific neuraminidase encoded by the pneumococcus genome, cleaving both a2-3-linked and a2-6-linked sialic acids. ${ }^{33-35}$ The less well-characterized $\mathrm{NanC}$ is more specific, cleaving only a2-3-linked sialic acids. ${ }^{36,37}$ As predicted, when sialic acids were labeled with biotin through PAL, we saw a decrease in biotinylation for cells treated with neuraminidase, as sialic acids had been removed (Figure 3a). Likewise, using GAL, we saw enrichment in biotinylation in neuraminidase-treated cells, as Gal and GalNAc residues were exposed upon sialic acid removal by NanA and NanC (Figure 3b). Notably, the neuraminidase-dependent change in GAL labeling appeared more dramatic than that seen for PAL labeling.

Satisfied we could use both PAL and GAL to differentially label cells treated with neuraminidases or left untreated, we next wanted to expand this method to include LCMS/MS for identification of neuraminidase protein substrates (Figure 2). In this way, neuraminidase substrates can be identified as those proteins that are enriched in untreated samples in PAL, or in neuraminidase-treated samples in GAL. While glycoproteomics techniques for identifying all sialylated proteins on cell surfaces are established, ${ }^{22}$ we expect this comparative proteomics strategy will selectively identify neuraminidase substrates, especially given that PAL-mediated labeling of cell surfaces suggests that not all sialylated glycans are completely desialylated after neuraminidase treatment (Figure 3a). Importantly, sialic acid produced by NanA and NanB activity has been shown to provide a carbon source for pneumococcus, ensuring growth. ${ }^{16,38}$ While this could mean neuraminidases are capable of cleaving every sialylated glycan on the cell surface, a study of NanC glycan specificity suggests that this neuraminidase is able to discriminate among underlying glycan structures. ${ }^{37}$ For this reason, we expect the method presented here will have a distinct 
advantage over methods identifying all sialylated proteins on the cell surface, and will enable us to build a candidate list of pneumococcal neuraminidase protein substrates that may function in pneumococcal infection of host cells.

To determine protein substrates using GAL, cells were treated with neuraminidases or not and then labeled by GAL. After cell lysis, labeled proteins were purified on streptavidin beads, and digested with trypsin. Resulting peptides were processed by LC-MS/MS followed by analysis using a label-free normalized spectral index quantitation method, SINQ, in which normalization of spectral counts allows for comparison between samples. ${ }^{23}$ The experiment was performed in triplicate; data are summarized in Supplementary Tables 1 and 2. While some proteins showed a neuraminidase-dependent enrichment in only one trial, a subset of proteins were enriched in all three trials. Focusing on proteins common to all three trials, we identified 58 proteins as substrates of NanA and 68 proteins as substrates of NanC (Figure 4a). Of these, 49 were common to both the NanA and NanC lists (Figure 4b). To ensure that the method was identifying proteins that could reasonably be expected to be substrates of an extracellular neuraminidase (i.e., proteins localized to the cell surface), we examined localization of hits. When evaluating each individual trial, we found a number of proteins were localized intracellularly (Supplementary Figure 2a). However, for proteins common to all three trials, the large majority of hits (95\% for NanA and $86 \%$ for NanC) were localized to the plasma membrane (Figure $4 \mathrm{c}$ ). In addition, most of the proteins identified in all three trials ( $88 \%$ for NanA and $80 \%$ for NanC) have been previously identified to be glycosylated (Supplementary Tables 1 and 2). We conclude that some proteins may be nonspecifically isolated in the streptavidin purification, and analysis of hits common to three trials provides a more accurate assessment of genuine neuraminidase substrates in hCMEC/D3 cells.

We next took a closer look at the substrate list from the GAL labeling experiment to determine whether identified proteins could be involved in pneumococcal infections. Indeed, at least four of the identified proteins (shown in black circles), ranging from low to high abundance, have had reported associations with pneumococcal infections (Figure 4d and 4e). Especially pleasing to us, one protein identified in all three trials was platelet endothelial cell adhesion molecule 1 (PECAM1), a putative adherence receptor for pneumococcus in brain endothelial cells. ${ }^{39}$ Indeed, while PECAM1 was isolated in both NanA- and NanC-treated samples, it was never identified in control, untreated samples (Supplementary Tables 1 and 2). Another protein of interest that appeared on the substrate list is endoglin (ENG). ENG functions in the TGF-beta signaling pathway, which can be utilized by pneumococcus to promote invasion in epithelial cells. ${ }^{40}$ In addition, we identified intracellular adhesion molecule 1 (ICAM1), which plays a critical role in inflammation of the brain during early stages of meningitis infection, and is upregulated by pneumococcus during in vitro models of infection. ${ }^{41}$ Finally, we identified basigin (BSG), which acts as an endothelial receptor in Neisseria meningitidis-induced bacterial meningitis. ${ }^{42}$ Therefore, proteins identified in our screen have strong potential to reveal new insights into neuraminidase-mediated pneumococcal infections.

Analogously to GAL, we next utilized PAL to identify proteins depleted in neuraminidasetreated cells compared to untreated cells. As with the GAL method, a number of proteins 
were enriched in control samples in only one of the three trials. However, 16 proteins were identified in all three trials for NanA, and 3 were identified for NanC (Figure 5a). Two proteins were common to both NanA and NanC (Figure 5b). Of the proteins identified in all three PAL trials, all were associated with the outer leaflet of the plasma membrane (Figure $5 c$ ), though examination of individual trials displayed a large percentage of intracellular proteins (Supplementary Figure 2b). Given the low number of hits common to all three trials and the high background observed in the PAL proteomics, we suggest GAL may be more robust in identifying neuraminidase substrates. The apparent superiority of GAL over PAL could be because the chemical oxidation method employed in PAL yields more nonspecific oxidation than the enzymatic oxidation method used in GAL. Also, in the GAL workflow only neuraminidase substrates are isolated for mass spectrometry, while in the PAL method both neuraminidase substrates and non-substrates are analyzed by mass spectrometry and the difference between the two must be discerned by analysis of spectral counts (Figure 2). Despite the fact that fewer proteins were identified by PAL, examination of the PAL protein list identified several possible candidates for mechanistic studies of pneumococcal infection. Along with BSG and ICAM1, multidrug-resistance protein 1 (P-glycoprotein, MRP1) and ephrin type-A receptor 2 (EPHA2) were identified in both NanA- and NanC-treated cells (Figure 5d and 5e). MRP1 deletion results in resistance to pneumococci-induced pneumonia in mice, and EPHA2 has been suggested to function as a receptor in some bacterial infections. ${ }^{43,44}$

A number of proteins were identified as neuraminidase substrates by both PAL and GAL methods (Figure 6a). However, unique targets were still identified in each method. While we did expect to observe some overlap between the PAL and GAL lists, there are multiple reasons why proteins may be identified in one scheme, but not the other. For example, if a protein bears multiple sialic acids, some of which are removed by the neuraminidase and some of which are not, then we might expect to see the protein identified in the GAL strategy (because a terminal Gal/GalNAc is revealed by neuraminidase treatment) but not in the PAL strategy (because the protein still bears a sialic acid after neuraminidase treatment). This reasoning may account for the significant number of proteins that were identified by GAL, but not PAL (Figure 6a). Conversely, the galactose oxidase that is used for labeling in the GAL strategy does not efficiently oxidize branched glycan structures. ${ }^{45}$ If, for a particular protein, the sialic acids removed by the neuraminidase are displayed only on branched glycans, then the protein may be identified by PAL, but not GAL. Our results indicate that PAL- and GAL-coupled comparative proteomics provide complementary approaches to identifying neuraminidase substrates, similar to the complementary value of PAL- and GAL-enabled proteomics in identifying total sialylated proteins. ${ }^{22}$

Upon establishing potential substrates for NanA and NanC, we next sought to verify proteins appearing on these lists. hCMEC/D3 cells were treated with NanA, NanC or buffer, then labeled with PAL or GAL, and lysed. Biotinylated proteins were purified with streptavidin, and analyzed by immunoblot using antibodies against three proteins that appeared on the GAL proteomics list. We were able to confirm the expected neuraminidase-dependent enrichment in GAL-labeled samples for all three proteins, PECAM1, ENG, and transferrin receptor protein 1 (TFRC) (Figure 6b). Moreover, each protein showed neuraminidasedependent depletion in PAL-labeled samples, even though none were identified by the PAL 
proteomics analysis. We examined two additional proteins, CUB domain-containing protein 1 (CDCP1), which appeared on the PAL proteomics list, and plexin-B2 (PLXNB2), which appeared on both lists. Again, neuraminidase-dependent changes in sialylation could be observed for both proteins, using both PAL and GAL labeling (Supplementary Figure 3). These results emphasize the complementary nature of the PAL and GAL labeling methods for identifying neuraminidase substrates.

In a confirmation method independent of chemoselective labeling, we also immunoprecipitated neuraminidase-treated and untreated lysates with MAL II, a lectin that specifically recognizes a 2-3 linked sialic acids, and found both ENG and TFRC were enriched in untreated samples versus those treated with NanA and NanC (Figure 6c). This result confirms that proteins identified in the proteomics analysis are bona fide neuraminidase substrates.

We performed gene ontology analysis using the Protein ANalysis THrough Evolutionary Relationships (PANTHER) classification system to identify biological processes and molecular functions that were enriched among the proteins that we identified as putative neuraminidase substrates (Figure 6d). ${ }^{46}$ The most highly enriched processes and functions ( $p$ $<0.05$ in the PANTHER overrepresentation test) are typical of membrane-associated receptors and transport proteins, consistent with the expected site of neuraminidase action. These results suggest that the action of pneumoccocal neuraminidases would be predicted to affect cell adhesion, membrane transport, and signal transduction processes.

Perhaps surprisingly, we did not observe distinct differences in putative protein substrates for NanA and NanC. As NanA is a less selective neuraminidase than NanC, we would expect to find proteins unique to NanA-treated samples that are not substrates of NanC. However, two proteins identified only in NanA-treated samples, TFRC and CDCP1, appeared to also be substrates of NanC when examined by our validation assays (Figure 6b and Supplementary Figure 3). Because NanA removes both a2-3- and a2-6-linked sialic acids, while NanC is selective for a2-3-linked sialic acids, this result suggests that we did not identify proteins that were exclusively sialylated with a2-6-linked sialic acids. We hypothesize that this may be due to a relatively low level of a2-6-linked sialic acid in hCMEC/D3 cells. Indeed, strong binding of the a2-3-linked sialic acid specific MAL II lectin to hCMEC/D3 cells compared to the a2-6-linked sialic acid specific Sambucus nigra lectin (SNA) suggests that hCMEC/D3 cells may express more a2-3-linked sialylated structures (Supplementary Figure 4). While there appears to be a high degree of overlap in substrates for pneumococcal neuraminidases, targets unique to either NanA or NanC might be identified in a cell line that expresses a higher level of a2-6-linked sialic acid or a different complement of sialylated structures.

We also assessed whether neuraminidase substrate proteins were sufficient to mediate adherence of pneumococcus to hCMEC/D3 cells. We chose to examine PECAM1, the putative receptor for pneumococcus adherence, ENG, and leukocyte surface antigen CD47, which has been shown to be upregulated in viral infection ${ }^{47,48}$ and is implicated in E. coli K1 meningitis infections. ${ }^{49}$ Cells were transfected with pools of siRNA against the protein of interest and silencing was verified by immunoblot (Supplementary Figure 5). Cells that 
had been transfected with siRNA against a protein of interest or scrambled siRNA were incubated with pneumococci for $30 \mathrm{~min}$. Cells were then lysed and plated in serial dilutions on blood agar plates, and the pneumococcal colonies that grew were counted. Though PECAM1 has been suggested to be a putative receptor of pneumococcal adherence, we observed only a slight, and not statistically significant, decrease in pneumococcus binding when PECAM1 was silenced (Figure 7a). Likewise, we saw no difference in adherence for cells in which CD47 had been silenced, and a slight, though not significant, decrease when endoglin had been silenced. These results contrast with global inhibition of glycosylation, where inhibition of GalNAc-type O-linked glycosylation or of sialylation were each sufficient to promote pneumococcus adherence (Figure 1a). Thus, it seems likely that multiple proteins participate in pneumococcus binding to host cells, and as such, silencing only one such protein produces too small of an effect to be measured. Indeed, two putative pneumococcal adherence receptors, PECAM1 and pIgR, have been shown to function in concert. ${ }^{39}$ Having a large candidate list, such as those produced in the PAL- and GALcoupled proteomics assays, could prove beneficial for identifying mechanisms through which pneumococcus enacts damage as it could highlight protein groups that function in concert to enable infections.

The proteomics analyses reported here identify substrates for purified recombinant neuraminidases. However, it would also be desirable to have a method that can be used to identify host glycoproteins that are desialylated by treatment with intact pneumococci, as these enzymes may exhibit different specificities when acting in the context of the pneumococcus. Therefore, to test whether this might be feasible, we assessed whether glycoproteins on the surface of host cells that were desialylated by pneumococci could be labeled using chemoselective chemistries. hCMEC/D3 cells were treated with pneumococci before labeling with GAL. Upon cell lysis and immunoblot analysis, we found that treatment with pneumococci led to an increase in biotinylation (Figure 7b). Therefore, not only can GAL detect changes in cell surface sialylation elicited with exogenously expressed proteins, but also those changes induced through the use of endogenously expressed enzymes in the bacteria. This result suggests that the comparative proteomics method described here could also be used to assess changes in host glycoprotein glycosylation induced by intact microorganisms.

Overall, we integrated existing chemoselective $\mathrm{e}^{21,22}$ and proteomics techniques ${ }^{22,23}$ to provide a comparative proteomics method for identifying protein substrates of neuraminidases. While a number of methods exist for determining glycan substrates of neuraminidases, ${ }^{37,50-57}$ to our knowledge, this is the first global assessment of protein substrates of neuraminidases. Given the roles of neuraminidases in contributing to pneumococcal pathogenesis through unveiling of protein receptors, ${ }^{14,16}$ this method provides a much-needed approach to identifying enzyme-linked mechanisms in disease. Indeed, this method could be applied to any pathogen in which neuraminidases play a key role in infection, such as Haemophilus influenzae, Vibrio cholerae, or Pseudomonas aeruginosa. ${ }^{58}$ Further analysis of identified neuraminidase substrates is predicted to shed light on important mechanisms in infection, and could reveal candidates for therapeutic targets. 


\section{Experimental Procedures}

\section{PAL labeling of cells}

hCMEC/D3 cells were trypsinized, washed in PBS, and treated with NanA, NanC, or buffer for $1 \mathrm{~h}$ in a $37{ }^{\circ} \mathrm{C}$ water bath. After washing in PBS containing $0.1 \%$ (w/v) BSA, cells were incubated in $1 \mathrm{mM}$ sodium periodate (Sigma) in PBS on ice for $30 \mathrm{~min}$ at $10 \times 10^{6}$ cells $\mathrm{mL}^{-1}$. Oxidation was quenched by addition of an equal volume of $1 \mathrm{mM}$ glycerol before washing with PBS. Cells were then incubated in PBS, pH 6.7, containing $5 \%$ (w/v) BSA, $250 \mu \mathrm{M}$ aminooxy-biotin (Biotium), and $10 \mathrm{mM}$ aniline (Sigma) to perform the oxime ligation. Incubation occurred for 90 minutes at $4{ }^{\circ} \mathrm{C}$ with constant rotation. For immunoblot analysis, cells were washed in PBS containing $0.1 \%$ BSA, lysed in RIPA buffer $(50 \mathrm{mM}$ Tris-HCl, pH 8.0, $150 \mathrm{mM} \mathrm{NaCl}, 1 \%$ (v/v) NP-40, $0.5 \%$ (w/v) sodium deoxycholate, $0.1 \%$ (w/v) sodium dodecyl sulfate (SDS), and protease inhibitor cocktail (Roche)), run on a $10 \%$ SDS-PAGE gel, and transferred overnight at $4{ }^{\circ} \mathrm{C}, 87 \mathrm{~mA}$ to a PVDF membrane.

\section{GAL labeling of cells}

hCMEC/D3 cells were trypsinized, washed in PBS, and treated with NanA, NanC, or buffer for $1 \mathrm{~h}$ in a $37^{\circ} \mathrm{C}$ water bath. Cells were then washed in PBS containing $0.1 \%(w / v)$ BSA. Oxidation and oxime ligation was performed by incubating cells in PBS, pH 6.7, containing $5 \%$ (w/v) BSA, $50 \mathrm{U} \mathrm{mL}^{-1}$ galactose oxidase from Dactylium dendoides (Sigma), $250 \mu \mathrm{M}$ aminooxy-biotin (Biotium), and $10 \mathrm{mM}$ aniline (Sigma) for $30 \mathrm{~min}$ in a $37^{\circ} \mathrm{C}$ water bath at $10 \times 10^{6}$ cells $\mathrm{mL}^{-1}$. For immunoblot analysis, cells were washed in PBS containing $0.1 \%$ BSA, lysed in RIPA buffer, run on a $10 \%$ SDS-PAGE gel, and transferred overnight at $4{ }^{\circ} \mathrm{C}$, $87 \mathrm{~mA}$ to a PVDF membrane.

\section{Proteomics analysis of PAL- and GAL-labeled samples}

hCMEC/D3 cells were harvested with trypsin, washed in PBS, and incubated with no sialidase (untreated) or with NanA or NanC for $1 \mathrm{~h}$ in a $37^{\circ} \mathrm{C}$ water bath. Cells were then washed in PBS containing $0.1 \%(\mathrm{w} / \mathrm{v}) \mathrm{BSA}$, and labeled using PAL or GAL as above. After labeling, cells were harvested at $1000 \mathrm{~g}$, washed in PBS containing $0.1 \%(\mathrm{w} / \mathrm{v}) \mathrm{BSA}$, and lysed ten minutes on ice in $50 \mathrm{mM}$ ammonium bicarbonate buffer containing $0.2 \%$ (w/v) RapiGest SF (Waters) and protease inhibitor (Roche). Cells were sonicated $15 \mathrm{~s}$ on, $15 \mathrm{~s}$ off at $20 \%$ power for $45 \mathrm{~s}$ total sonication time. Lysates were then centrifuged at 20,000g for 10 min at $4{ }^{\circ} \mathrm{C}$. Proteins were quantified by BCA assay kit (Pierce) and $400-800 \mu \mathrm{g}$ of protein was incubated with $50 \mu \mathrm{L}$ streptavidin agarose and rotated overnight at $4{ }^{\circ} \mathrm{C}$. Beads were pelleted and washed three times in lysis buffer, four times in PBS containing $2 \mathrm{M}$ urea, and 4 times in PBS. For trypsin digest, beads were first resuspended in digestion buffer (6 M urea, $100 \mathrm{mM}$ Tris (pH 8.0), 2M thiourea, $50 \%$ (v/v) trifluoroethanol, and $0.1 \%$ (w/v) RapiGest $\mathrm{SF}$ ) and incubated at $37^{\circ} \mathrm{C}$ for $30 \mathrm{~min}$. Tris(2-carboxyethyl)phosphine (TCEP) was then added to a final concentration of $10 \mathrm{mM}$ and beads were incubated at $37^{\circ} \mathrm{C}$ for another 30 min. Next, iodoacetamide was added to a final concentration of $12 \mathrm{mM}$ and beads were incubated at room temperature in the dark for $30 \mathrm{~min}$. Finally, beads were diluted 1:10 in $100 \mathrm{mM}$ Tris, $\mathrm{pH} 8.0$ with $10 \mathrm{mM} \mathrm{CaCl}_{2}$ and sequence grade modified trypsin (Promega) was added to a final concentration of $10 \mu \mathrm{g} \mathrm{mL}-1$. Trypsin digest was allowed to occur overnight in a shaking $37^{\circ} \mathrm{C}$ incubator. After incubation, an additional $10 \mathrm{mg} \mathrm{mL}^{-1}$ trypsin 
was added to digests and allowed to proceed for another $2 \mathrm{~h}$ at $37^{\circ} \mathrm{C}$ with shaking. Supernatants were then removed from the beads, and reactions were stopped by addition of trifluoroacetic acid to $\mathrm{pH}<2(\sim 0.5 \%(\mathrm{v} / \mathrm{v})$ of total supernatant volume). Supernatants were incubated $45 \mathrm{~min}$ at $37^{\circ} \mathrm{C}$, then centrifuged at $20,000 \mathrm{~g}$ for $10 \mathrm{~min}$ at room temperature. Samples were submitted to the UTSW Proteomics Core for analysis, as follows.

Digested samples were cleaned using solid-phase extraction with Oasis HLB plates (Waters) and were then analyzed by LC-MS/MS, using a Q Exactive mass spectrometer (Thermo Electron) coupled to an Ultimate 3000 RSLC-Nano liquid chromatography system (Dionex). Samples were injected onto a $180 \mu \mathrm{m}$ i.d., $15-\mathrm{cm}$ long column packed in-house with a reverse-phase material, ReproSil-Pur C18-AQ, $3 \mu \mathrm{m}$ resin (Dr. Maisch GmbH, AmmerbuchEntringen, Germany), and eluted with a gradient from 1-28\% buffer B over $40 \mathrm{~min}$. Buffer A contained $2 \%(\mathrm{v} / \mathrm{v})$ acetonitrile (ACN) and $0.1 \%$ formic acid in water, and buffer B contained $80 \%(\mathrm{v} / \mathrm{v}) \mathrm{ACN}, 10 \%(\mathrm{v} / \mathrm{v})$ trifluoroethanol, and $0.08 \%$ formic acid in water. The mass spectrometer operated in positive ion mode with a source voltage of $2.4 \mathrm{kV}$, capillary temperature of $250{ }^{\circ} \mathrm{C}$, and S-lens RF level at $50.0 \%$. MS scans were acquired at 70,000 resolution and up to $20 \mathrm{MS} / \mathrm{MS}$ spectra were obtained for each full spectrum acquired at 17,500 resolution using higher-energy collisional dissociation (HCD) for ions with charge $\geq 2$. Dynamic exclusion was set for $7 \mathrm{~s}$.

Raw MS data files were converted to a peak list format and analyzed using the central proteomics facilities pipeline (CPFP), version 2.0.3. ${ }^{59,60}$ Peptide identification was performed using the X!Tandem ${ }^{61}$ and open MS search algorithm (OMSSA) ${ }^{62}$ search engines against the human protein database from Uniprot, with common contaminants and reversed decoy sequences appended. ${ }^{63}$ Fragment and precursor tolerances of 20 ppm and 0.1 Da were specified, and three miscleavages were allowed. Carbamidomethylation of Cys was set as a fixed modification and oxidation of Met was set as a variable modification. Label-free quantitation of proteins across samples was performed using SINQ normalized spectral index Software. ${ }^{23}$

For analysis of PAL proteomics, proteins in untreated samples were sorted by spectral counts, and only those with counts $>3$ were analyzed. The ratio of the untreated over NanAor NanC-treated MIC SIn values were calculated, and those above 3 were considered hits. Similarly, for GAL proteomics, proteins in NanA- and Nan-C treated samples were sorted by spectral counts, and only those with counts $\geq 3$ were analyzed. The ratio of the Nan-A or NanC-treated over untreated MIC SIn was calculated, and those above 3 were considered hits in GAL. Resulting lists from each trial were then compared to determine proteins that occurred in all three trials. Cellular localization and annotation of glycosylation of hits was determined using the UniProt database. Data are presented in Supplementary Tables 1 and 2.

To determine function of protein hits, PANTHER analysis was used (pantherdb.org; PANTHER version 10.0, released 2015-05-15). ${ }^{46}$ Proteins appearing in all three trials of the PAL or GAL strategy were assessed using the PANTHER Overrepresentation Test (released 2015-04-30) and PANTHER GO-Slim biological process or PANTHER GO-Slim molecular function dataset (reference list: Homo Sapiens). Results with a $p<0.05$ are presented in Figure 6d. 


\section{Supplementary Material}

Refer to Web version on PubMed Central for supplementary material.

\section{Acknowledgments}

We thank the NIH (R01GM090271) and the Welch Foundation (I-1686) for funding. JEM was supported by a postdoctoral fellowship from The Hartwell Foundation. Support for proteomics analysis was provided by the Cancer Prevention Research Institute of Texas (R1121 and RP120613) and the Welch Foundation (I-1850). We thank A. Camilli (Tufts University) for providing pneumococcus and training and thank B. Weksler (Weill Cornell Medical College), P.-O. Couraud (INSERM), and I. Romero (The Open University) for sharing hCMEC/D3 cells. We thank Drs. S. Krishnamurthy, N. Nischan, and A. Wands for comments on the manuscript.

\section{References}

1. von Itzstein M. The war against influenza: discovery and development of sialidase inhibitors. Nat Rev Drug Discov. 2007; 6:967-974. [PubMed: 18049471]

2. Bosch AATM, Biesbroek G, Trzcinski K, Sanders EAM, Bogaert D. Viral and bacterial interactions in the upper respiratory tract. PLoS Pathog. 2013; 9:e1003057. [PubMed: 23326226]

3. Warwas ML, Yeung JHF, Indurugalla D, Mooers AO, Bennet AJ, Moore MM. Cloning and characterization of a sialidase from the filamentous fungus, Aspergillus fumigatus. Glycoconj J. 2010; 27:533-548. [PubMed: 20652740]

4. Frasch AC. Functional diversity in the trans-sialidase and mucin families in Trypanosoma cruzi. Parasitol Today. 2000; 16:282-286. [PubMed: 10858646]

5. Jedrzejas MJ. Pneumococcal virulence factors: structure and function. Microbiol Mol Biol Rev. 2001; 65:187-207. [PubMed: 11381099]

6. Li N, Ren A, Wang X, Fan X, Zhao Y, Gao GF, Cleary P, Wang B. Influenza viral neuraminidase primes bacterial coinfection through TGF- $\beta$-mediated expression of host cell receptors. Proc Natl Acad Sci U S A. 2015; 112:238-243. [PubMed: 25535343]

7. Chang YC, Uchiyama S, Varki A, Nizet V. Leukocyte inflammatory responses provoked by pneumococcal sialidase. MBio. 2012; 3:e00220-11. [PubMed: 22215570]

8. King SJ. Pneumococcal modification of host sugars: a major contributor to colonization of the human airway? Mol Oral Microbiol. 2010; 25:15-24. [PubMed: 20331791]

9. Bridy-Pappas AE, Margolis MB, Center KJ, Isaacman DJ. Streptococcus pneumoniae: description of the pathogen, disease epidemiology, treatment, and prevention. Pharmacotherapy. 2005; 25:1193-1212. [PubMed: 16164394]

10. Mook-Kanamori BB, Geldhoff M, van der Poll T, van de Beek D. Pathogenesis and pathophysiology of pneumococcal meningitis. Clin Microbiol Rev. 2011; 24:557-591. [PubMed: 21734248]

11. Pilishvili T, Lexau C, Farley MM, Hadler J, Harrison LH, Bennett NM, Reingold A, Thomas A, Schaffner W, Craig AS, et al. Sustained reductions in invasive pneumococcal disease in the era of conjugate vaccine. J Infect Dis. 2010; 201:32-41. [PubMed: 19947881]

12. Hava DL, Camilli A. Large-scale identification of serotype 4 Streptococcus pneumoniae virulence factors. Mol Microbiol. 2002; 45:1389-1406. [PubMed: 12207705]

13. Pettigrew MM, Fennie KP, York MP, Daniels J, Ghaffar F. Variation in the presence of neuraminidase genes among Streptococcus pneumoniae isolates with identical sequence types. Infect Immun. 2006; 74:3360-3365. [PubMed: 16714565]

14. King SJ, Hippe KR, Weiser JN. Deglycosylation of human glycoconjugates by the sequential activities of exoglycosidases expressed by Streptococcus pneumoniae. Mol Microbiol. 2006; 59:961-974. [PubMed: 16420364]

15. Marion C, Limoli DH, Bobulsky GS, Abraham JL, Burnaugh AM, King SJ. Identification of a pneumococcal glycosidase that modifies O-linked glycans. Infect Immun. 2009; 77:1389-1396. [PubMed: 19139197] 
16. King SJ, Hippe KR, Gould JM, Bae D, Peterson S, Cline RT, Fasching C, Janoff EN, Weiser JN. Phase variable desialylation of host proteins that bind to Streptococcus pneumoniae in vivo and protect the airway. Mol Microbiol. 2004; 54:159-171. [PubMed: 15458413]

17. Manco S, Hernon F, Yesilkaya H, Paton JC, Andrew PW, Kadioglu A. Pneumococcal neuraminidases A and B both have essential roles during infection of the respiratory tract and sepsis. Infect Immun. 2006; 74:4014-4020. [PubMed: 16790774]

18. Chen GY, Chen X, King S, Cavassani KA, Cheng J, Zheng X, Cao H, Yu H, Qu J, Fang D, et al. Amelioration of sepsis by inhibiting sialidase-mediated disruption of the CD24-SiglecG interaction. Nature Biotech. 2011; 29:428-435.

19. Uchiyama S, Carlin AF, Khosravi A, Weiman S, Banerjee A, Quach D, Hightower G, Mitchell TJ, Doran KS, Nizet V. The surface-anchored NanA protein promotes pneumococcal brain endothelial cell invasion. J Exp Med. 2009; 206:1845-1852. [PubMed: 19687228]

20. Banerjee A, Van Sorge NM, Sheen TR, Uchiyama S, Mitchell TJ, Doran KS. Activation of brain endothelium by pneumococcal neuraminidase NanA promotes bacterial internalization. Cell Microbiol. 2010; 12:1576-1588. [PubMed: 20557315]

21. Zeng Y, Ramya TNC, Dirksen A, Dawson PE, Paulson JC. High-efficiency labeling of sialylated glycoproteins on living cells. Nat Methods. 2009; 6:207-209. [PubMed: 19234450]

22. Ramya T, Weerapana E, Cravatt BF, Paulson JC. Glycoproteomics enabled by tagging sialic acidor galactose-terminated glycans. Glycobiology. 2013; 23:211-221. [PubMed: 23070960]

23. Trudgian DC, Ridlova G, Fischer R, Mackeen MM, Ternette N, Acuto O, Kessler BM, Thomas B. Comparative evaluation of label-free SINQ normalized spectral index quantitation in the central proteomics facilities pipeline. Proteomics. 2011; 11:2790-2797. [PubMed: 21656681]

24. Weksler BB, Subileau EA, Perrière N, Charneau P, Holloway K, Leveque M, Tricoire-Leignel H, Nicotra A, Bourdoulous S, Turowski P, et al. Blood-brain barrier-specific properties of a human adult brain endothelial cell line. FASEB J. 2005; 19:1872-1874. [PubMed: 16141364]

25. Elbein AD, Tropea JE, Mitchell M, Kaushal GP. Kifunensine, a potent inhibitor of the glycoprotein processing mannosidase I. J Biol Chem. 1990; 265:15599-15605. [PubMed: 2144287]

26. Kuan SF, Byrd JC, Basbaum C, Kim YS. Inhibition of mucin glycosylation by aryl-N-acetyl-alphagalactosaminides in human colon cancer cells. J Biol Chem. 1989; 264:19271-19277. [PubMed: 2509474]

27. Rillahan CD, Antonopoulos A, Lefort CT, Sonon R, Azadi P, Ley K, Dell A, Haslam SM, Paulson JC. Global metabolic inhibitors of sialyl- and fucosyltransferases remodel the glycome. Nat Chem Biol. 2012; 8:661-668. [PubMed: 22683610]

28. Huang J, Byrd JC, Yoon WH, Kim YS. Effect of benzyl-alpha-GalNAc, an inhibitor of mucin glycosylation, on cancer-associated antigens in human colon cancer cells. Oncol Res. 1992; 4:507-515. [PubMed: 1284381]

29. Huet G, Kim I, de Bolos C, Lo-Guidice JM, Moreau O, Hemon B, Richet C, Delannoy P, Real FX, Degand P. Characterization of mucins and proteoglycans synthesized by a mucin-secreting HT-29 cell subpopulation. J Cell Sci. 1995; 108:1275-1285. [PubMed: 7622610]

30. Wands AM, Fujita A, McCombs JE, Cervin J, Dedic B, Rodriguez AC, Nischan N, Bond MR, Mettlen M, Trudgian DC, et al. Fucosylation and protein glycosylation create functional receptors for cholera toxin. eLife. 2015; 4:e09545. [PubMed: 26512888]

31. Gahmberg CG, Andersson LC. Selective radioactive labeling of cell surface sialoglycoproteins by periodate-tritiated borohydride. J Biol Chem. 1977; 252:5888-5894. [PubMed: 69628]

32. Gahmberg CG, Hakomori SI. External labeling of cell surface galactose and galactosamine in glycolipid and glycoprotein of human erythrocytes. J Biol Chem. 1973; 248:4311-4317. [PubMed: 4711609]

33. Xu G, Potter JA, Russell RJM, Oggioni MR, Andrew PW, Taylor GL. Crystal structure of the NanB sialidase from Streptococcus pneumoniae. J Mol Biol. 2008; 384:436-449. [PubMed: 18835278]

34. Gut H, King SJ, Walsh MA. Structural and functional studies of Streptococcus pneumoniae neuraminidase B: An intramolecular trans-sialidase. FEBS Lett. 2008; 582:3348-3352. [PubMed: $18775704]$ 
35. Xu G, Li X, Andrew PW, Taylor GL. Structure of the catalytic domain of Streptococcus pneumoniae sialidase NanA. Acta Crystallogr Sect F. 2008; 64:772-775.

36. Xu G, Kiefel MJ, Wilson JC, Andrew PW, Oggioni MR, Taylor GL. Three Streptococcus pneumoniae sialidases: Three different products. J Am Chem Soc. 2011; 133:1718-1721. [PubMed: 21244006]

37. Parker RB, McCombs JE, Kohler JJ. Sialidase specificity determined by chemoselective modification of complex sialylated glycans. ACS Chem Biol. 2012; 7:1509-1514. [PubMed: 22704707]

38. Burnaugh AM, Frantz LJ, King SJ. Growth of Streptococcus pneumoniae on human glycoconjugates is dependent upon the sequential activity of bacterial exoglycosidases. J Bacteriol. 2008; 190:221-230. [PubMed: 17981977]

39. Iovino F, Molema G, Bijlsma JJE. Platelet endothelial cell adhesion molecule-1, a putative receptor for the adhesion of Streptococcus pneumoniae to the vascular endothelium of the blood-brain barrier. Infect Immun. 2014; 82:3555-3566. [PubMed: 24914219]

40. Malipiero U, Koedel U, Pfister W, Fontana A. Bacterial meningitis: the role of transforming growth factor-Beta in innate immunity and secondary brain damage. Neurodegener Dis. 2007; 4:43-50. [PubMed: 17429218]

41. Thornton J, McDaniel LS. THP-1 monocytes up-regulate intercellular adhesion molecule 1 in response to pneumolysin from Streptococcus pneumoniae. Infect Immun. 2005; 73:6493-6498. [PubMed: 16177322]

42. Bernard SC, Simpson N, Join-Lambert O, Federici C, Laran-Chich MP, Maïssa N, BouzinbaSégard H, Morand PC, Chretien F, Taouji S, et al. Pathogenic Neisseria meningitidis utilizes CD147 for vascular colonization. Nat Med. 2014; 20:725-731. [PubMed: 24880614]

43. Schultz MJ, Wijnholds J, Peppelenbosch MP, Vervoordeldonk MJ, Speelman P, van Deventer SJ, Borst P, van der Poll T. Mice lacking the multidrug resistance protein 1 are resistant to Streptococcus pneumoniae-induced pneumonia. J Immunol. 2001; 166:4059-4064. [PubMed: 11238654]

44. Subbarayal P, Karunakaran K, Winkler AC, Rother M, Gonzalez E, Meyer TF, Rudel T. EphrinA2 receptor (EphA2) is an invasion and intracellular signaling receptor for Chlamydia trachomatis. PLoS Pathog. 2015; 11:e1004846. [PubMed: 25906164]

45. Schlegel RA, Gerbeck CM, Montgomery R. Substrate specificity of D-galactose oxidase. Carbohydr Res. 1968; 7:193-199.

46. Mi H, Poudel S, Muruganujan A, Casagrande JT, Thomas PD. PANTHER version 10: expanded protein families and functions, and analysis tools. Nucl Acids Res. 2016; 44:D336-42. [PubMed: 26578592]

47. Wang JH, Kwon HJ, Jang YJ. Rhinovirus enhances various bacterial adhesions to nasal epithelial cells simultaneously. Laryngoscope. 2009; 119:1406-1411. [PubMed: 19434681]

48. Mlacha SZK, Peret TCT, Kumar N, Romero-Steiner S, Hotopp JCD, Ishmael N, Grinblat-Huse V, Riley DR, Erdman DD, Carlone GM, et al. Transcriptional adaptation of pneumococci and human pharyngeal cells in the presence of a virus infection. BMC Genomics. 2013; 14:378. [PubMed: 23742656]

49. Mittal R, Gonzalez-Gomez I, Prasadarao NV. Escherichia coli K1 promotes the ligation of CD47 with thrombospondin-1 to prevent the maturation of dendritic cells in the pathogenesis of neonatal meningitis. J Immunol. 2010; 185:2998-3006. [PubMed: 20675593]

50. Corfield AP, Higa H, Paulson JC, Schauer R. The specificity of viral and bacterial sialidases for a(2-3)- and a(2-6)-linked sialic acids in glycoproteins. Biochim Biophys Acta. 1983; 744:121126. [PubMed: 6301560]

51. Cabezas JA, Calvo P, Eid P, Martin J, Perez E, Reglero A, Hannoun C. Neuraminidase from influenza virus A (H3N2): specificity towards several substrates and procedure of activity determination. Biochim Biophys Acta. 1980; 616:228-238. [PubMed: 7213637]

52. Rogerieux F, Belaise M, Terzidis-Trabelsi H, Greffard A, Pilatte Y, Lambré CR. Determination of the sialic acid linkage specificity of sialidases using lectins in a solid phase assay. Anal Biochem. 1993; 211:200-204. [PubMed: 7686353] 
53. Onodera S. A microplate assay for sialidase activity using plant lectin binding to Nacetyllactosamine. Biol Pharm Bull. 1994; 17:29-33. [PubMed: 7511958]

54. Chokhawala HA, Yu H, Chen X. High-throughput substrate specificity studies of sialidases by using chemoenzymatically synthesized sialoside libraries. Chembiochem. 2007; 8:194-201. [PubMed: 17195254]

55. Indurugalla D, Watson JN, Bennet AJ. Natural sialoside analogues for the determination of enzymatic rate constants. Org Biomol Chem. 2006; 4:4453-4459. [PubMed: 17268638]

56. Li Y, Cao H, Yu H, Chen Y, Lau K, Qu J, Thon V, Sugiarto G, Chen X. Identifying selective inhibitors against the human cytosolic sialidase NEU2 by substrate specificity studies. Mol Biosyst. 2011; 7:1060-1072. [PubMed: 21206954]

57. Cao H, Li Y, Lau K, Muthana S, Yu H, Cheng J, Chokhawala HA, Sugiarto G, Zhang L, Chen X. Sialidase substrate specificity studies using chemoenzymatically synthesized sialosides containing C5-modified sialic acids. Org Biomol Chem. 2009; 7:5137-5145. [PubMed: 20024109]

58. Lewis AL, Lewis WG. Host sialoglycans and bacterial sialidases: a mucosal perspective. Cell Microbiol. 2012; 14:1174-1182. [PubMed: 22519819]

59. Trudgian DC, Thomas B, McGowan SJ, Kessler BM, Salek M, Acuto O. CPFP: a central proteomics facilities pipeline. Bioinformatics. 2010; 26:1131-1132. [PubMed: 20189941]

60. Trudgian DC, Mirzaei H. Cloud CPFP: a shotgun proteomics data analysis pipeline using cloud and high performance computing. J Proteome Res. 2012; 11:6282-6290. [PubMed: 23088505]

61. Craig R, Beavis RC. TANDEM: matching proteins with tandem mass spectra. Bioinformatics. 2004; 20:1466-1467. [PubMed: 14976030]

62. Geer LY, Markey SP, Kowalak JA, Wagner L, Xu M, Maynard DM, Yang X, Shi W, Bryant SH. Open mass spectrometry search algorithm. J Proteome Res. 2004; 3:958-964. [PubMed: 15473683]

63. Elias JE, Gygi SP. Target-decoy search strategy for increased confidence in large-scale protein identifications by mass spectrometry. Nat Methods. 2007; 4:207-214. [PubMed: 17327847] 
a

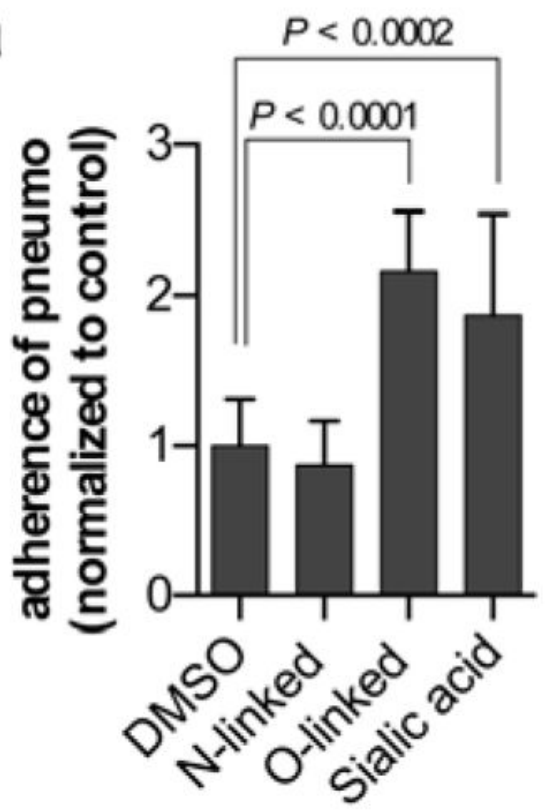

b

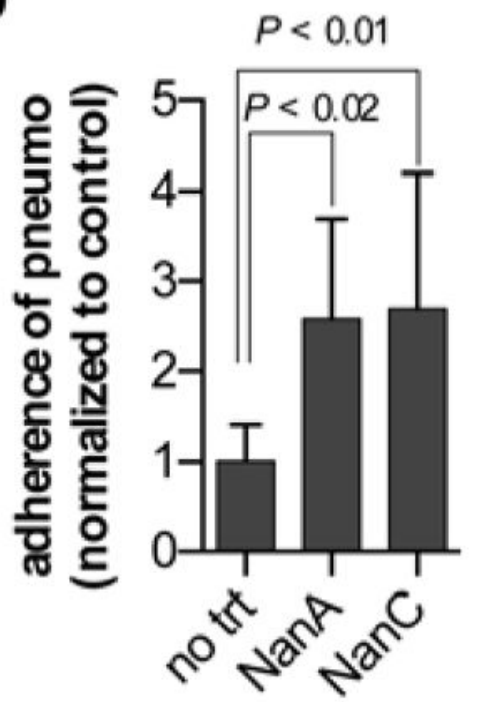

Figure 1. Adherence of pneumococcus to hCMEC/D3 cells is altered by changes in glycosylation (a) N-linked glycan maturation, O-linked glycan synthesis, and sialylation of cell surface glycans (sialic acid) were inhibited in hCMEC/D3 cells prior to incubation with pneumococcus. Colony forming units were determined for each condition and compared to DMSO-treated control cells. (b) hCMEC/D3 cells were pre-treated with NanA, NanC, or buffer (untreated) before incubation with pneumococcus. Colony forming units were determined for each treatment and compared to untreated cells. Error bars represent standard deviation of three trials. 
a PAL
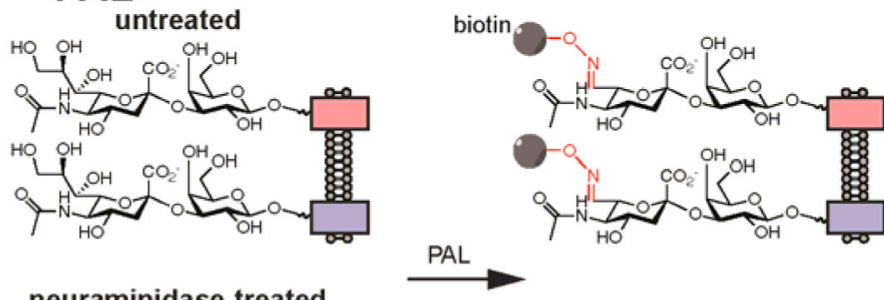

1. Lysis

2. Streptavidi enrichment

Substrate ID

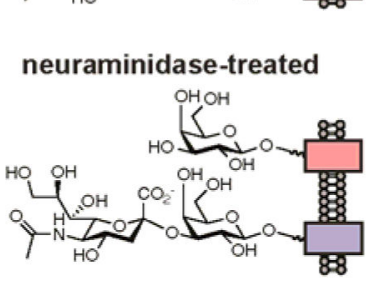
3. LC-MS/MS
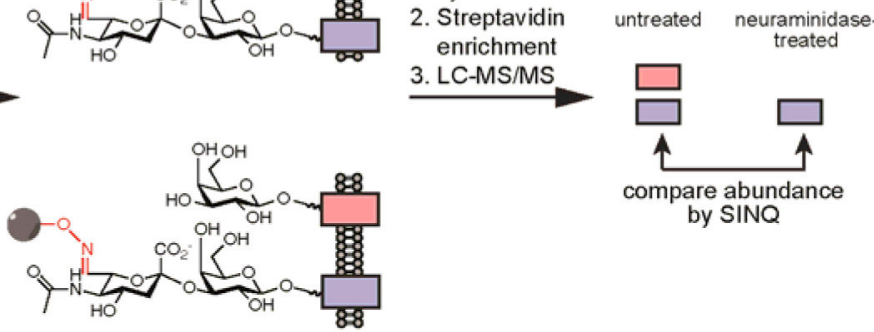

compare abundance
by SINQ bINQ

\section{b GAL}
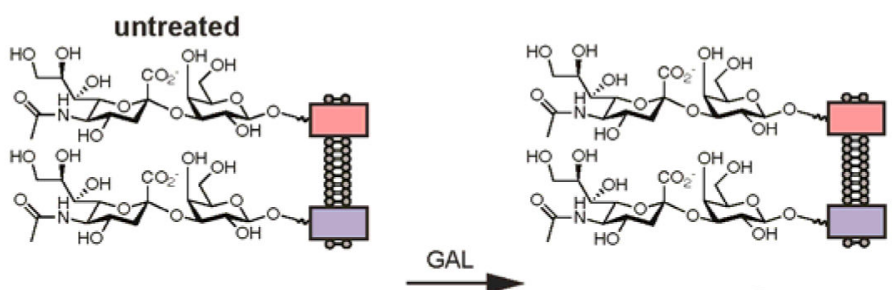
1. Lysis
2. Streptavidin enrichment 3. LC-MS/MS

untreated neuraminidase-

neuraminidase-treated
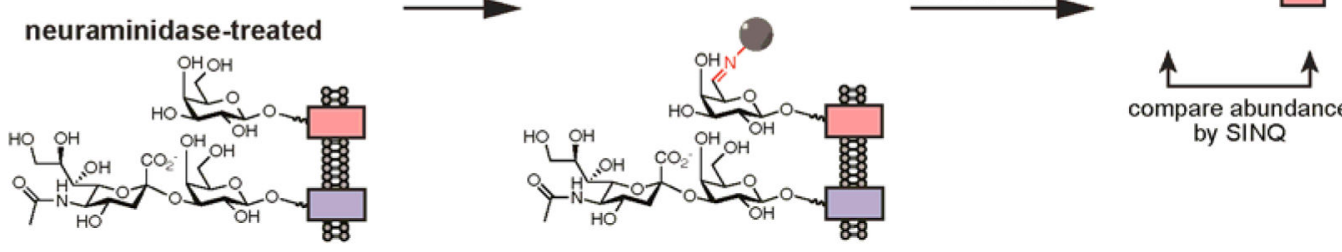
by SINQ

Figure 2. Schematic of GAL- and PAL-coupled proteomics to identify neuraminidase substrates Cells left untreated or treated with neuraminidase are labeled with biotin (gray ball) using PAL (a) or GAL (b). Substrate proteins are depicted in pink and non-substrate proteins in blue. Cells are then lysed, and biotinylated proteins are enriched through streptavidin purification. Proteins bound to beads are washed, digested with trypsin, and identified by LC-MS/MS. Substrates of neuraminidases can be identified by comparative proteomics analysis (SINQ) as those enriched in untreated samples for PAL or neuraminidase-treated samples for GAL. 
a

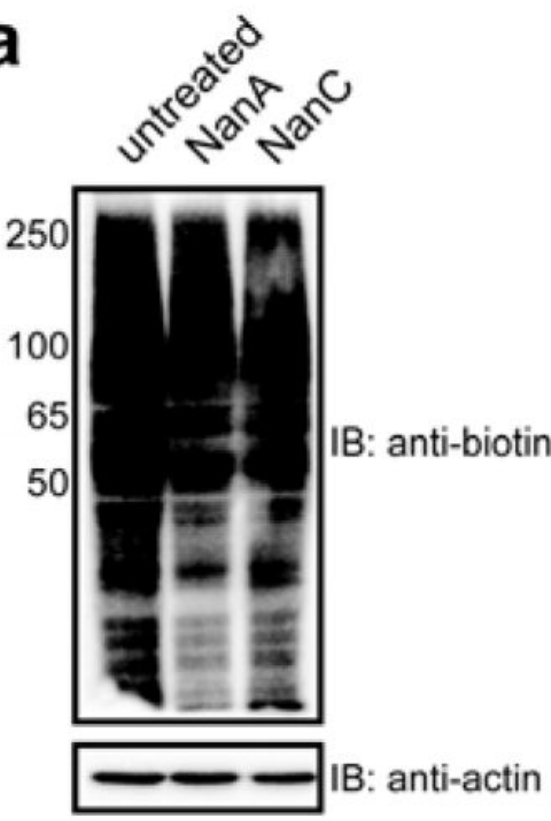

b

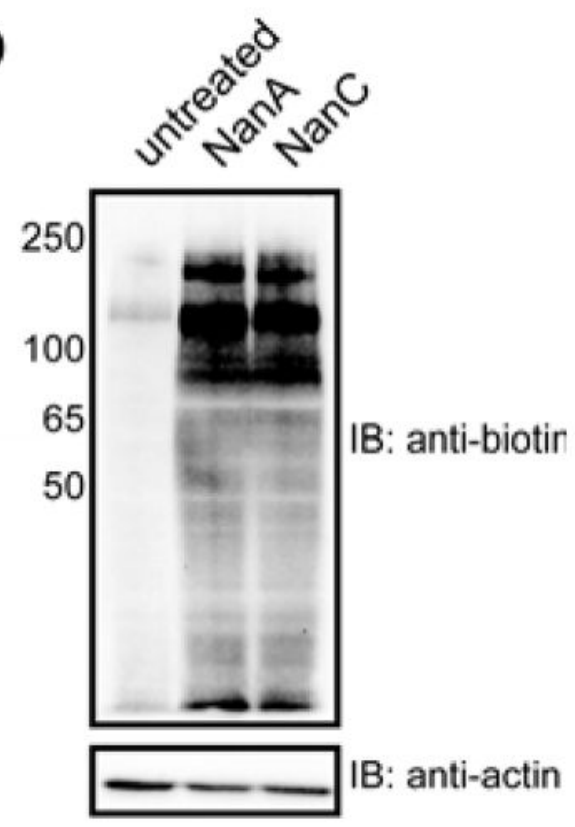

Figure 3. PAL and GAL label glycoproteins in a neuraminidase-dependent manner Immunoblots of lysates from hCMEC/D3 cells left untreated or treated with NanA or NanC and then labeled with aminooxy-biotin using (a) PAL, or (b) GAL. 
a

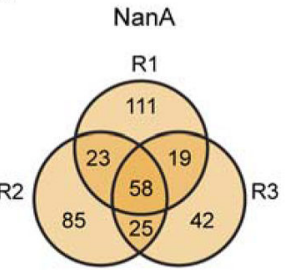

R2

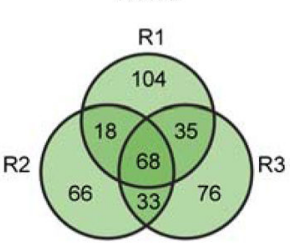

d

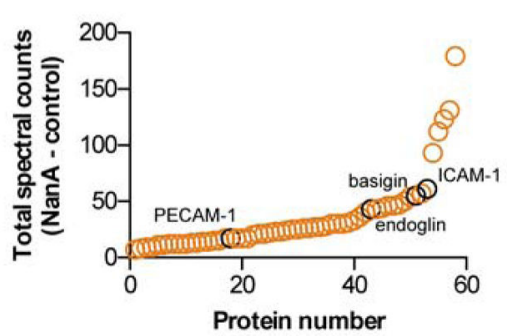

b

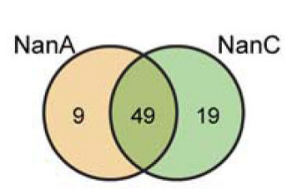

e

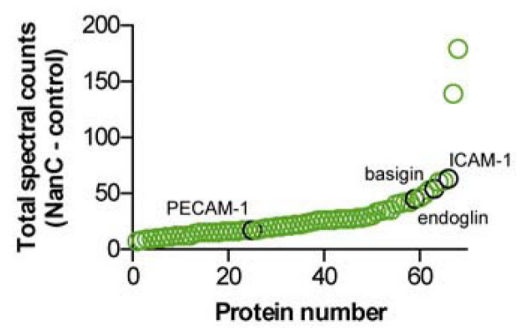

Figure 4. GAL-coupled proteomics reveals a large number of neuraminidase protein substrates (a) Comparison of number of proteins identified in NanA- and NanC-treated cells in three biological replicates. (b) Overlap in number of proteins identified in all three trials between NanA- and NanC-treated samples. (c) Localization of proteins identified for NanA and NanC is primarily at the membrane. (d) Total spectral counts (sum of counts in NanAtreated samples minus sum in untreated samples) for proteins identified in all three trials for NanA. Proteins denoted with black circles have previously reported associations with bacterial infections. (e) Total spectral counts (sum of counts in NanC-treated samples minus sum in untreated samples) for proteins identified in all three trials for NanC. Proteins denoted with black circles have previously reported associations with bacterial infections. 
a

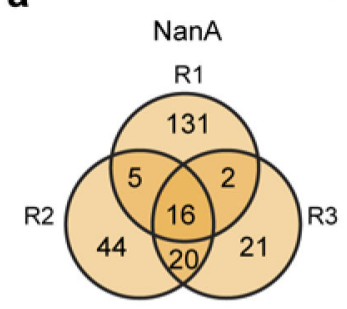

C

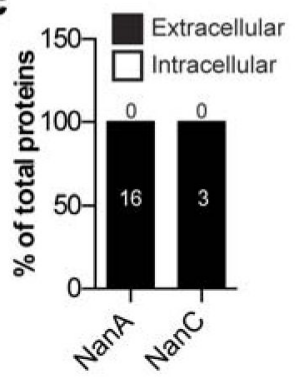

PAL Results

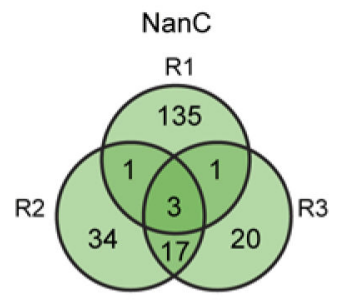

d

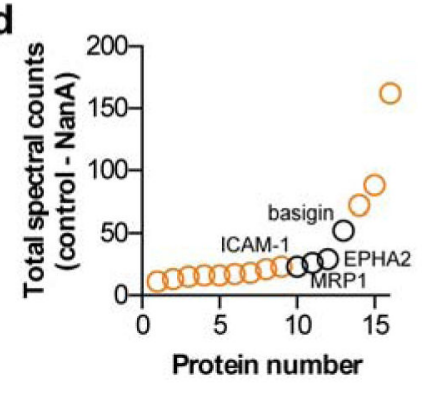

b

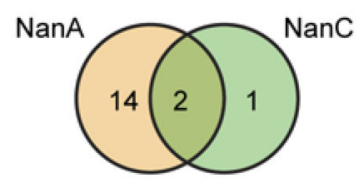

e

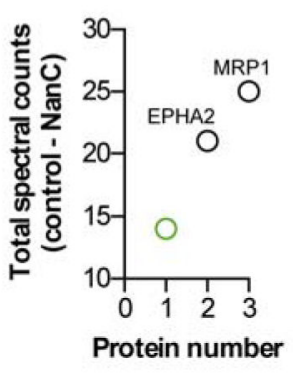

Figure 5. PAL-coupled proteomics reveals fewer substrates than GAL-coupled proteomics (a) Comparison of number of proteins identified in NanA- and NanC-treated cells in three biological replicates. (b) Overlap in number of proteins identified in all three trials between NanA- and NanC-treated samples. (c) Localization of proteins identified for NanA and NanC is solely at the membrane. (d) Total spectral counts (sum of counts in untreated samples minus sum of counts in NanA-treated samples) for proteins identified in all three trials for NanA. Proteins denoted with black circles have previously reported associations with bacterial infections. (e) Total spectral counts (sum of counts in untreated samples minus sum of counts in NanC-treated samples) for proteins identified in all three trials for NanC. Proteins denoted with black circles have previously reported associations with bacterial infections. 
a

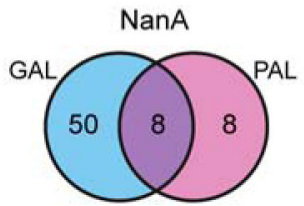

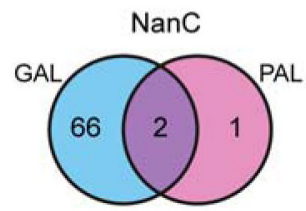

c

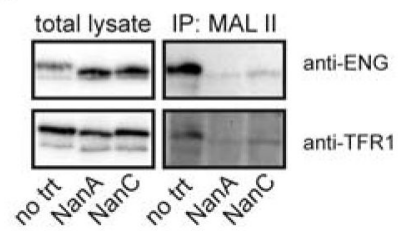

PAL; PAL; total lysate streptavidin total lysate streptavidin

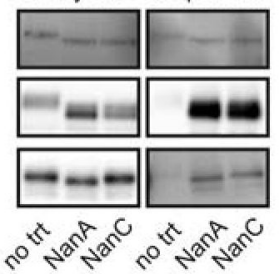

IB: anti-PECAM

anti-ENG

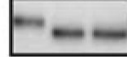

$-\ldots-\infty$

anti-TFR1

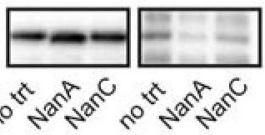

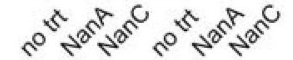

d

biological process

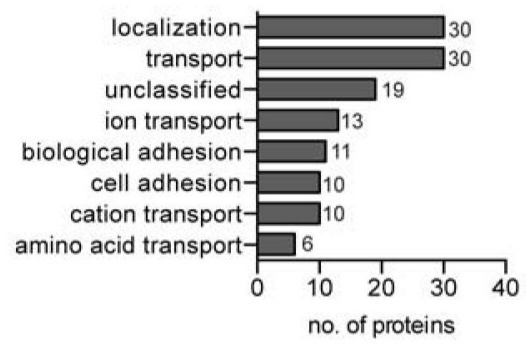

molecular function

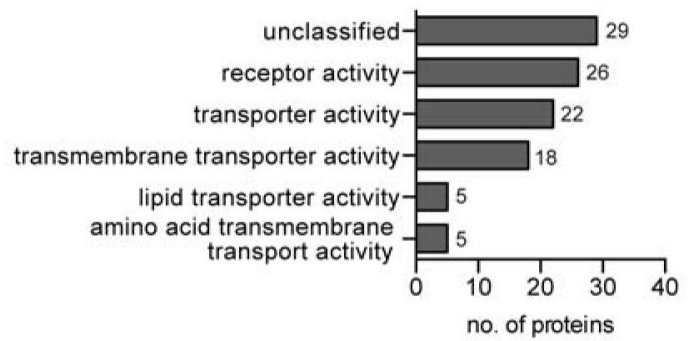

Figure 6. Glycoproteins identified in GAL- and PAL-coupled proteomics verified as neuraminidase substrates

(a) Overlap of proteins identified in GAL and PAL approaches for NanA-treated samples (left) and NanC-treated samples (right). (b) Immunoblots of proteins of interest from neuraminidase-treated or untreated GAL- (left) or PAL-labeled (right) cells after streptavidin purification. (c) Immunoblots of proteins of interest from neuraminidase-treated or untreated cells immunoprecipitated with the a2-3-linked sialic acid specific lectin MAL II-biotin followed by streptavidin purification. (d) Biological processes and molecular functions enriched among identified proteins as determined by the PANTHER overrepresentation test. Results have a $p$-value $>0.05$ when compared to the Homo sapiens reference list (PANTHER version 10.0, released 2015-05-15). ${ }^{46}$ 
a

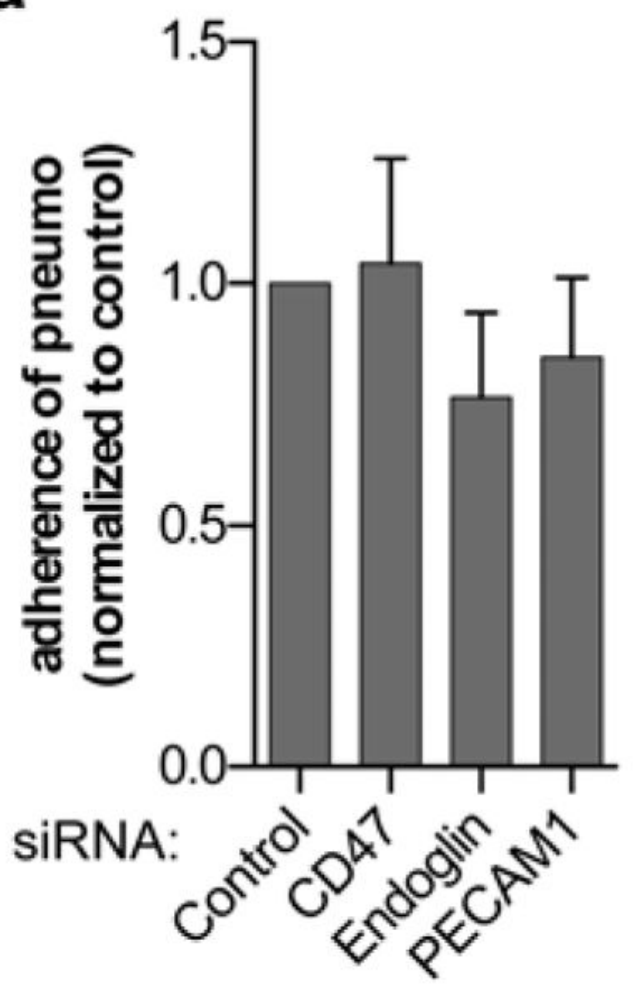

b

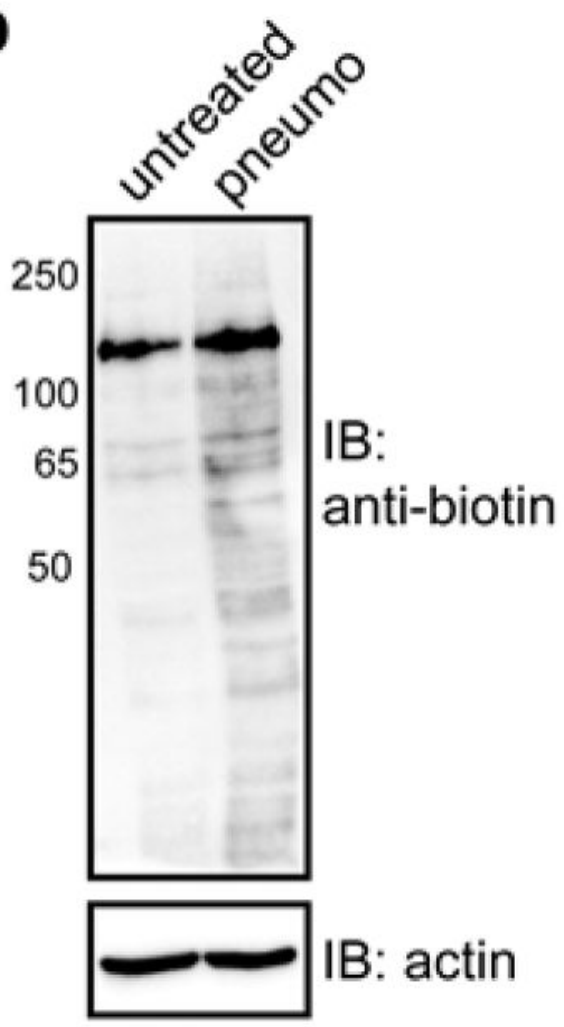

Figure 7. Pneumococci desialylate multiple glycoprotein substrates that may act in concert to enable infection

(a) Adherence of pneumococci to hCMEC/D3 cells is unchanged in cells with indicated proteins silenced using siRNA compared to cells transfected with scrambled siRNA. (b) hCMEC/D3 cells treated with pneumococci or not were labeled with biotin using GAL. 